\title{
The Synthesis and Regulation of Micronutrients in Rice Grains
}

\section{Bo Peng"}

College of Life Sciences and Institute for Conservation and Utilization of Agro-bioresources in Dabie Mountains, Xinyang Normal University, Xinyang 464000, China

\section{Dongyan Kong}

College of Life Sciences and Institute for Conservation and Utilization of Agro-bioresources in Dabie Mountains, Xinyang Normal University, Xinyang 464000, China

\section{Yu Peng}

School of Science and Technology, Xinyang University, Xinyang 464000, China

\section{Cheelo Dimuna}

College of Life Sciences and Institute for Conservation and Utilization of Agro-bioresources in Dabie Mountains, Xinyang Normal University, Xinyang 464000, China

\section{Lulu He}

College of Life Sciences and Institute for Conservation and Utilization of Agro-bioresources in Dabie Mountains, Xinyang Normal University, Xinyang 464000, China

\section{Qingqing Xin}

College of Life Sciences and Institute for Conservation and Utilization of Agro-bioresources in Dabie Mountains, Xinyang Normal University, Xinyang 464000, China

\section{Yue Jiang}

College of Life Sciences and Institute for Conservation and Utilization of Agro-bioresources in Dabie Mountains, Xinyang Normal University, Xinyang 464000, China

\section{Yanfang Sun}

College of Life Sciences and Institute for Conservation and Utilization of Agro-bioresources in Dabie Mountains, Xinyang Normal University, Xinyang 464000, China

\section{Ruihua Pang}

College of Life Sciences and Institute for Conservation and Utilization of Agro-bioresources in Dabie Mountains, Xinyang Normal University, Xinyang 464000, China

\section{Xiaohua Song}

Xinyang Academy of Agricultural Sciences, Xinyang 464000, China

\section{Huilong Li}

Xinyang Academy of Agricultural Sciences, Xinyang 464000, China

\section{Juan Peng}

Xinyang Station of Plant Projection and Inspection, Xinyang 464000, China

\section{Ziyue Liu}

College of Life Sciences and Institute for Conservation and Utilization of Agro-bioresources in Dabie Mountains, Xinyang Normal University, Xinyang 464000, China

\section{Yuchen Liu}

College of International Education, Xinyang Normal University, Xinyang 464000, China

\section{Shizhi Song}

College of International Education, Xinyang Normal University, Xinyang 464000, China

\section{Hongyu Yuan}

College of Life Sciences and Institute for Conservation and Utilization of Agro-bioresources in Dabie Mountains, Xinyang Normal University, Xinyang 464000, China 


\section{Abstract}

Amino acids, vitamins and lipids are the important micronutrients in rice grains. Their synthesis and regulation have important effects on the normal growth and development in rice seeds. This review has mainly summarized the new advances in the synthesis and regulation of amino acids, vitamins and lipids in rice grains. Simultaneously, the challenges of the synthesis and accumulation of the micronutrients in rice grains were also discussed. This review provides important information for genetic improvement of grain quality in rice and, potentially, other staple cereals.

Keywords: Micronutrients; Amino acids; Vitamins; Lipids; Synthesis; Regulation; Rice.

(a) (4) CC BY: Creative Commons Attribution License 4.0

\section{Introduction}

Rice (Oryza sativa L.) is one of the most important food crops providing about $25 \%$ of the energy demand for more than 3 billion people in the world. Therefore, rice is an extremely important source of energy and nutrients for human beings [1]; [2]; [3]. In the past few decades, rice yield has steadily increased and reached a higher level. However, the overall quality of rice grain is not optimistic in the market [4]. The quality traits of rice grain are quite complicated, and it is generally considered that rice grain quality includes the grain appearance and milling, eating, cooking and nutritional qualities. Simultaneously, rice grain quality directly affects the market price of rice in the world which in turn is closely related to human nutrition and health [5]. With the continuous increase of global population and the gradual improvement of the quality of life, the demand for high quality rice will continue to grow strongly in the future [6]; [7].

The main nutrients in rice grain are starch and storage proteins. These two account for more than $90 \%$ of the dry weight of rice grains [8]; [9]. The main types of starch available in rice grains include amylose and amylopectin whose structure and relative content have important effects on many quality traits of rice. For instance,the relative abundance of amylose and amylopectin has a correlated effect on the appearance quality, processing quality, cooking quality, nutritional quality and eating quality of rice grains [10]. Storage protein content and amino acid content and their relative equilibrium are the most important factors determining the grain nutritional quality in rice [3]; [11], and the storage protein content will also affect its eating quality, processing quality and appearance quality in rice grain [12]. Storage protein provides about $15 \%$ of human protein sources and it could be classified into glutenin, prolamin, globulin and albumin according to the different separation and extraction methods [8]. The micronutrients in rice grain mainly include amino acids, vitamins and lipids, and their composition and relative content in rice grains have a certain influence on the rice grain qualities, such as eating quality, appearance quality, nutritional quality, cooking quality and processing quality [13]; [14]. Therefore, the composition and relative content of nutrients in rice grain are closely related to human health.

In the past decade, important progress has been made in the genetic improvement of nutrients in rice grain [6]; [15]; [16]; [17]. A large number of genes have been isolated and cloned to regulate the synthesis and degradation of nutrients such as amino acids, lipids and vitamins in rice [15]; [18]; [19]. The nutrients in rice grain are closely related to the quality traits of rice. Therefore, to elucidate the synthesis, regulatory network and genetic basis of amino acids, lipids and vitamins in rice has great values both on theory and practice for genetic improvement of grain quality in the future. Here, we focus on the recent new advances in the synthesis and regulation of micronutrients such as amino acids, lipids and vitamins in rice grains. Furthermore, the strategies for genetic improvement of the nutrients in rice are also discussed and this will provide an important reference for the genetic improvement of the grain quality of crops and the cultivation of new varieties.

\section{The Synthesis and Regulation of Amino Acids in Rice Grains}

Amino acids play a very important role in plant growth and metabolism [20]. The contents of lysine and tryptophan in grains are low, and they are the first and second restricted essential amino acids in rice respectively. The content of lysine is also a relatively low essential amino acid in other main grain crops (such as corn, wheat, etc.), thus lysine is considered as the first limiting amino acid in cereal crops [21]; Ufaz and Galili [22]. In order to meet the needs of human nutrition balance, it is necessary to improve the protein content and the relative content of amino acids in rice grain. Through the aspartic acid metabolism pathway, not only lysine but also the other three essential amino acids, methionine, threonine and isoleucine could be synthesized in rice [23]. Aspartic kinase (AK), dihydropyridine dicarboxylic acid synthase (DHDPS) and lysine ketoglutarate reductase/yeast aminoate dehydrogenase (LKR/SDH) are the three key enzymes in aspartic acid metabolism pathway [24]. Among them, AK and DHDPS play a feedback inhibitory role in aspartic acid metabolism pathway. AK catalyzes the first step of lysine synthesis pathway, while DHDPS catalyzes the first step of dihydropyridine dicarboxylic acid branching. At the same time, lysine and threonine can feedback the inhibition of AK while lysine is the regulatory inhibitory factor of DHDPS Vidal, et al. [25]. When the content of lysine in grains is high, the activity of LKR/SDH will increase correspondingly, which will promote the degradation process of lysine in vivo and lead to the low content of lysine in grains [26]; [27]. A large number of studies based on aspartic acid metabolism pathway have indicated that if the expression of $A K$ and DHDPS genes was promoted or the expression of $L K R / S D H$ genes and 13-kDa prolamine genes was inhibited, the lysine content in rice plants was significantly increased [28]; [29]. If the lysine-rich exogenous protein was specifically expressed in rice grains or the $R L R H 1$ and $R L R H 2$ genes were overexpressed, the lysine content in rice grains could also be significantly increased [30]; [31]. Therefore, the specific quantitative expression of the key genes in the lysine metabolism pathway can greatly enhance the lysine content in rice grains. 
Tryptophan and phenylalanine, are essential amino acids which can not be synthesized by the human and animal bodies.They play an important role in the growth, development and metabolism of both human and animal [32]. Tryptophan and phenylalanine belong to aromatic amino acids which are the precursors of various secondary metabolites in rice. They are closely related to the growth and development of rice and even the rice grain quality [33]. Therefore, increasing the content of tryptophan and phenylalanine in rice grain is of great significance to the improvement of nutritional quality of rice. Cuurently, there are many studies on increasing the content of lysine and methionine in rice grain while those aiming at trying to increase the content of tryptophan and phenylalanine in rice are relatively less [34].

Aromatic amino acids belong to the metabolic pathway of shikimic acid in plants, bacteria and fungi (Fig. 1), and they share a common precursor, branching acid. Various alpha-subunit-related genes of feedback-insensitive oaminobenzoic acid synthase (AS) have been used in the genetic improvement of tryptophan in crops [33]; [35]. In order to improve the content of tryptophan, a large number of corresponding mutants had been used, but it was difficult to find the mutants with significant changes in phenylalanine content [36]; [37]. The dehydratase ADT/PDT encoded by Mtr 1 could catalyze the last step of phenylalanine biosynthesis in the overexpressing of transgenic plants [38], and the contents of tryptophan and phenylalanine increased significantly, suggesting that oaminobenzoic acid synthetase and ADT/PDT dehydratase play a key role in regulating the metabolism of tryptophan and phenylalanine in rice grains.

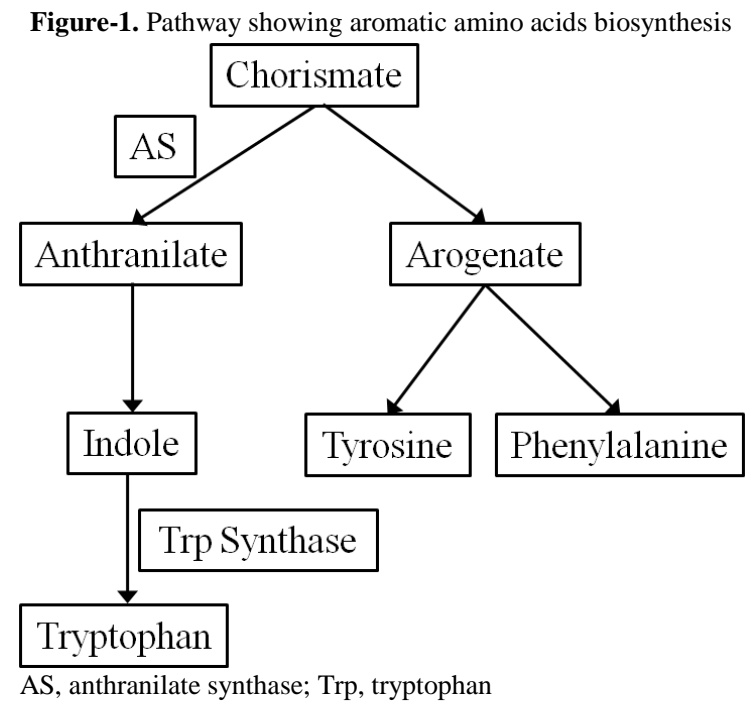

Cysteine and methionine are important amino acids that constitute proteins and the synthesis of the former (cysteine) can enhance the antioxidant stress ability in plants. Most other sulfur metabolites are directly or indirectly derived from cysteine, thus cysteine is at the center of sulfur metabolism in plants [39]; [40]. Serine acyltransferase and 3-phosphoglycerate dehydrogenase are two rate-limiting enzymes in the process of cysteine biosynthesis. Hydrogen sulfide and O-acetylserine (OAS) finally react with cysteine synthase (OAS-TL) to form cysteine [40]. Two rate-limiting enzymes (serine acyltransferase and glycerol 3-phosphate dehydrogenase) strictly regulate the catalytic reaction of cysteine synthesis resulting in the low content of cysteine. The synthesis of cysteine synthase complex (CSC), using cysteine synthase (OAS-TL) and serine acetyltransferase (SAT) will be more effective in regulating the process of cysteine biosynthesis [41]. Methionine is an essential amino acid that humans and animals can't synthesize themselves. Methionine as one of the essential protein source provides active methyl groups for the body, and it can also be converted into cysteine in vivo [42]; [43];. The lack of methionine can cause a variety of hazards to humans and livestock, and long-term consumption of foods with low methionine content may lead to many diseases. For example, methionine deficiency in livestock's diet will lead to the decrease of wool in sheep, milk production and meat quality in cows. The absence of methionine human diet will affect the absorption and utilization of other related amino acids by the body [44]. Therefore, increasing the content of methionine has been one of the important goals pursued by plant geneticists and breeders [33]. The expression of serine acetyltransferase gene was driven by a promoter with ubiquitin, which increased methionine and cysteine in rice by 1.4 times and 2.4 times respectively [45]. Simultaneously, the contents of leucine and valine also increased significantly, indicating that methionine could be transformed into isoleucine in rice. Therefore, the use of genetic engineering strategy can significantly improve the content of essential amino acids in rice grain, and then improve the nutritional quality of rice.

\section{The Synthesis and Regulation of Lipid in Rice Grains}

Lipid, including fat and phospholipids, is a very important nutrient in rice grains. It is mainly distributed in the aleurone layer outside the embryo and endosperm of rice seeds where a complex of lipids and amylose in rice grains form $\mathrm{Xu}$, et al. [46]; Goufo, et al. [47]. At present, many QTLs have been located in rice genome and are closely related to lipids in their grains. However, the isolation and cloning of QTL related genes are rare Shao and Bao [48]. Fatty acid oxidase (LOX) is an important factor leading to the decline of rice nutritional quality, because it can 
catalyze the oxidation of lipids [23]. Both $L O X-1, L O X-2, L O X-3$ and $r 9-L O X-1$ in rice genome encode fatty acid oxidase [49]; [50]. It was found that $L O X-2, L O X-3$ or $r 9-L O X-1$ could inhibit the degradation of fatty acids. The degradation of $\beta$-carotene in golden rice could be effectively reduced by decreasing the expression of $L O X-3$ or $r 9$ $L O X-1$.

Very long chain polyunsaturated fatty acid (VLCPUFAs) and long chain polyunsaturated fatty acid (LCPUFA) are essential regulatory substances for the synthesis and transport of cholesterol and eicosanoid [51]. They are the main components of nerve cells (such as the brain and retina) [52]; which in turn affect the development and health of the human body. Ultra long chain polyunsaturated fatty acids can be synthesized through different pathways (e.g. $\omega$-6 metabolic pathway or $\omega-3$ metabolic pathway) [53]. Therefore, proteins or enzymes encoded by many genes can improve the level of ultra long chain polyunsaturated fatty acids such as FAD3, D5 prolongase gene, $\omega-3$ fatty acid desaturase gene, ${ }^{\Delta} 8$-desaturase gene and ${ }^{\Delta} 5$-desaturase gene [54]; [55]; [56]; [57]. FAD3 protein can catalyze the synthesis of $\alpha$-linolenic acid in rice grains, and then it can be used to increase the content of $\alpha$-linolenic acid in rice. $\alpha$-linolenic acid is an important precursor of long-chain $\omega 3$-unsaturated fatty acids, and its content in rice grain is relatively low. If the FAD3 gene is over-expressed, the content of $\alpha$-linolenic acid in rice grains can be greatly increased [58]. Although three FAD3 genes have been cloned in rice, it is unclear how these genes play a role in increasing the concentration of $\alpha$-linolenic acid in rice grains.

Oil protein is abundant in the oil body of plant seeds and can be used to regulate the content of fat in seeds. The over-expression of soybean oil body protein gene was driven by rice endosperm-specific promoter, the fat content in transgenic rice grains increased by more than $36 \%$, however, the total triglyceride fatty acid content did not change significantly [59]. Rice oil contains a large number of antioxidant substances such as oryzanol, lecithin, tocopherol and fertility trienol, which are beneficial to human health Choi, et al. [60]. The expression of GmFAD3-1 and $O S F A D 3$ genes was driven by rice embryo-specific promoter $R E G$, which resulted in a significant increase in $\alpha$ linolenic acid content in rice embryos and aleurone layers. The increased $\alpha$-linolenic acid is located in the sn- 2 position of triglyceride and is easily digested and absorbed by human body Yin, et al. [61]. Previous studies have shown that OsLTP36 encoded a lipid transporter gene in rice, and if OsLTP36 gene was down regulated, it would seriously affect the development of rice seeds, and could significantly reduce the lipid content in rice grains [62]; [63]. The FAD3 gene of Brassica napus was specifically expressed in rice, which could significantly increase the content of C18: 3 fatty acids and improve the nutritional quality of rice [58]. At present, although important progress has been made in the study of lipid metabolism and some genes related to lipid metabolism in rice have also been isolated and cloned, how to regulate lipid metabolism pathway in rice grains remains to be further studied.

\section{The Synthesis and Regulation of Vitamin in Rice Grains}

The low levels of vitamin A in the endosperm of rice grains cause more than 250 million people in the world who rely on rice as their staple food to experience varying levels of vitamin A deficiency, which could lead to immune system-related diseases, or even permanent blindness [64]. Carotenoids, mainly beta-carotenoids are easily converted into the precursors to synthesize vitamin A in humans [65]. At present, genes related to the pathway of carotenoid biosynthesis have been successfully isolated and cloned, and these genes are widely found in bacteria, fungi and plants [66]. Although rice can synthesize carotenoids in leaves, some enzymes in the pathway of carotenoid synthesis are not expressed in the endosperm of rice. After genetic engineering modification, rice can produce a lot of $\beta$-carotenoids in endosperm and form yellow "Golden Rice". The first generation of Golden Rice contains $1.6 \mu \mathrm{g}$ carotenoid per gram of dry weight of rice grain, which is equivalent to $100 \mu \mathrm{g}$ of retinol in $300 \mathrm{~g}$ of rice per day, and it will greatly alleviate the symptoms of vitamin A deficiency in children [67]; [68]. By using maize octahydrolycopene synthase gene (psy) instead of narcissus octahydrolycopene synthase gene in the second generation of Golden Rice, the carotenoid content in rice grain could reach $37 \mu \mathrm{g}^{-g^{-1}}$ [69]. It is feasible to increase the $\beta$-carotene level in different indica and japonica rice varieties [66]; [70], but there is still a long way to go for the commercialization of golden rice.

The content of thiamine or vitamin B1 in rice grains is relatively low, with only about $18 \%$ of the recommended daily consumption content, thus vitamin B1 should be supplemented by other ways. Rice aleurone layer and embryo (containing more thiamine than endosperm) has been removed in the process of grain polishing, which leads to the further decrease of thiamine content in milled rice (mainly endosperm). Therefore, the lack of thiamine may cause beriberi in the population with milled rice as the only staple food [71]. In recent years, important progress has been made in the biosynthetic pathway of thiamine in plants [72]; [73], the synthesis of thiamine occurs in plastids. Under the action of thiamine phosphate (TMP) synthase, the two groups of pyrimidine and thiazole are condensed to form TMP, and hydrolysis of TMP into thiamine in cytoplasm and conversion to thiamine pyrophosphate (TPP). TPP produced by this pathway can bind to the precursor of thiamine biosynthetic gene and interfere with its gene expression, and regulating the ribosomal switch by RNA sequence. Interestingly, TPP ribosomal switch sequence located in the 3'-UTR region of THIC gene plays a negative regulatory role in thiamine biosynthesis [74]; [75]. The contents of TMP and TPP increased significantly in the leaves of plants with overexpression of THIC gene [76]. However, further research is needed to increase the content of thiamine in the endosperm of rice grains.

Folic acid, also known as vitamin B9 plays an important role in human growth and development. Lack of folic acid in diet can lead to many diseases [77]. Many genes have been found to be involved in the synthesis and metabolism of folic acid in plants such as GTP cyclase gene $(G T P C H I)$, aminodeoxysynthase gene $(A D C)$, hydroxymethyl dihydropurine gene $(H M D H P)$, pyrophosphokinase gene $(H P P K)$ and dihydrocharidase synthase gene $(D H P)$ etc [78]; [79]. The over-expression of $A D C S$ gene will increase the content of benzoic acid in plants, but the increase of benzoic acid will seriously inhibit the biosynthesis of folic acid, and ultimately lead to the decrease of 
folic acid content [80]. There was no significant change in folic acid content in transgenic plants with the overexpression of GTPCHI gene, but it should increase the folic acid content in transgenic plants to 50-100 times than the original level when GTPCHI gene and ADCS gene were overexpressed at the same time [81]. Using the promoter from maize driven barley gene HPPK/DHPS to express in rice, it was found that the content of folic acid in transgenic rice seeds reached about 1.5 times of the original level [77]. Therefore, through the strategy of metabolic engineering, using the genes related to folic acid biosynthesis pathway in close related species of rice, it can also increase the content of folic acid in rice grains.

Vitamin $\mathrm{E}$ is a compound that includes tocopherol and reproductive trienol family members in eight forms of fat-soluble antioxidants. As an important part of human defense, vitamin E provides anti-oxidative damage protection, thus reducing the occurrence of various diseases. The overexpression of hydroxyl pyruvate dioxygenase gene (HPPD) from Arabidopsis thaliana has no obvious effect on the content of tocopherol, but it can produce more reproductive tocotrienols. Finally, the activity of vitamin E in rice grains increased significantly [82]. In rice genome, at least 7 genes, such as MT1/2, OsHGGT, OsMPBQ, OsHPPD, OsTC, OsHPT and OsTMT, have been found to be involved in the synthesis of vitamin E in rice seeds [83]; [84]. The expression level of OsTMT gene and its alleles was closely related to the content of alpha-tocopherol in rice grains [65]. Overexpression of $O s H P T$ gene can also increase the content of vitamin E components [85]. If $G E$ gene mutation occurs, it can enhance the expression of genes related to vitamin $E$ synthesis and metabolism, and then increase the content of vitamin $E$ in rice grains [83]. Interestingly, over-expression of GmTMT2a resulted in a significant increase in the content of alphatocopherol [86], which provided a new idea for increasing the content of alpha-tocopherol in rice grains.

Vitamin C, also known as ascorbic acid, is an important water-soluble vitamin with antioxidant, antiatherosclerosis, improving immunity and anti-cancer properties. However, its content in rice grains is also very low [87]. Humans are unable to synthesize this important vitamin due to the lack of glycosolactone oxidase. Although the pathway of vitamin $\mathrm{C}$ biosynthesis and its associated genes are relatively clear in plants but there is little known about them in monocotyledonous plants especially in rice [33]. If the over-expression of the genes from Arabidopsis thaliana involved in vitamin $\mathrm{C}$ synthesis and metabolism (such as AtGGP, AtGDH, AtGME, AtGPP, AtGMP and AtGalLDH), the content of vitamin $\mathrm{C}$ in rice grains could be significantly increased [88]. Mutations in the genes related to vitamin $\mathrm{C}$ synthesis and metabolism in rice will not only reduce the content of vitamin $\mathrm{C}$ in the grains [89], but also have an important impact on the stress resistance and the development of the whole plant in rice. Therefore, in order to solve the problem of low vitamin content in rice grains, an increase in the vitamin content of rice seeds and improved nutritional quality, rice must make full use of its genes. To achieve this, we can also consider the introduction of exogenous genes, metabolic engineering, genetic engineering and other modern technical methods.

\section{The Challenge and Prospect of Nutrient Improvement in Rice Grains}

The nutrients of rice grains include starch, storage proteins, lipids, amino acids, vitamins and so on are improved by genetic engineering or metabolic engineering [90]. To produce the best functional proteins or enzymes driven by appropriate promoters of the target gene and promote the synthesis of macronutrients and micronutrients in rice grains, the growth and development of plants and other metabolic pathways need not to be affected [91]. At present, the biosynthetic pathway and regulation mechanism of some nutrients in rice are still unclear, this in turn limits the application of genetic engineering or metabolic engineering for genetic improvement in rice. Nutrients in rice grains have been researched extensively and important progress has been made by using the strategies of multi-omics (proteomics, metabolomics, transcriptome, etc.). For example, some undesirable allergic proteins improved by genetic engineering in rice can be screened by proteomics [92], and multiple metabolites related to abiotic stress resistance and nutritional starvation can be accurately quantified and identified in rice [93]; [94]. It is especially helpful to promote the accumulation of beneficial nutrients in seeds, which will be of great significance to improve human health.

At present, gas chromatography mass spectrometry (GC-MS), liquid chromatography mass spectrometry (LCMS), capillary electrophoresis mass spectrometry (CE-MS), X-ray fluorescence spectrometry (XRF), energy dispersive X-ray spectrometry (EDX), scanning electron microscopy (SEM) and transmission electron microscopy (TEM), as well as single cell imaging (SCIM) have developed rapidly in recent years [1]. All of these will provide new technical solutions for further research on the synthesis and accumulation of nutrients in rice grains. However, genetic improvement in rice grains is still challenging in view of the multiple steps in the process of simultaneous action of multiple genes on biosynthetic pathways or the multiple traits of multiple biosynthetic pathways in rice. Although many genes related to the synthesis of nutrients in rice grains have been revealed, functional genes are often directly or indirectly regulated by other genes [33], and there is a common phenomenon that the genes may have multiple effects. Therefore, functional genes that can be widely used ingrain nutrient genetics improvement and breeding are relatively rare in rice. In order to promote the synthesis and accumulation of nutrients in rice grains, these functional genes often need to develop specific, tissue-specific or inducible promoters to drive their expression.

In recent decades, genome editing technology based on sequence-specific nucleases (SSNs) has developed rapidly, and has become one of the most effective new tools for rice genetic improvement [95]; [96]. Particular attention should be given to CRISPR/Cas9 technology, which has many advantages: (1) Editing target genes accurately; (2) There is no need for hybridization and backcross, and it is convenient and fast; (3) No need for large capital investment; (4) Individuals without selection markers can be obtained [96]; [97]. Therefore, CRISPR/Cas9 technology has been widely used in rice nutrition biosynthesis and metabolism researches [98]; [99]. Therefore, genome editing technology, represented by CRISPR/Cas9 technology will play a more and more important role in 
the process of grain nutrition genetic improvement and breeding of new varieties in rice. This will eventually greatly accelerate the genetic improvement of rice grain quality.

\section{Acknowledgements}

This work was financially supported by National Natural Science Foundation of China (U1604110, U1404319, 31600992, 31801332), Key Project of Science and Technology in Henan Province (182102110442), Major Science and Technology Project in Henan Province (121100110200), Key Project of Innovation and Entrepreneurship for Undergraduate in Henan Province (S201810477004), Nanhu Scholars Program for Young Scholars of XYNU (2016054), Student Research Fund Project of XYNU (2018-DXS-066), Key Scientific Research Projects of Universities in Henan Province (19A180030) and Institute for Conservation and Utilization of Agro-bioresources in Dabie Mountains.

\section{References}

[1] Tian, Z. X., Qian, Q., and Liu, Q. Q., 2009. "Allelic diversities in rice starch biosynthesis lead to a diverse array of rice eating and cooking qualities." Proceedings of the National Academy of Sciences, vol. 106, pp. 21760-21765.

[2] Peng, B., Kong, H. L., and Li, Y. B., 2014. "Osaap6 functions as an important regulator of grain protein content and nutritional quality in rice." Nature Communications, vol. 5, p. 4847.

[3] Peng, B., Sun, Y. F., and Chen, B. Y., 2017. "Research progress of fragrance gene and its application in rice." Chinese Bulletin of Botany, vol. 52, pp. 797-807.

[4] Qiang, X. T., Zhao, C. F., and Zhao, L., 2016. "Analysis of genetic variation and population structure of starch synthesis related genes in indica rice cultivar." Jiangsu Journal of Agriculture Sciences, vol. 32, pp. 241-249.

[5] Peng, B., Kong, D. Y., and Pang, R. H., 2017. "Detection and application of functional markers of badh2 gene from different fragrant rice varieties in southern henan." Southwest China Journal of Agricultural Science, vol. 30, pp. 1693-1699.

[6] Myint, K. M., Arikit, S., and Wanchans, 2012. "A pcr-based marker for a locus conferring the aroma in myanmar rice (oryza sativa 1)." Theoretical and Applied Genetics, vol. 125, pp. 887-896.

[7] Ootsuka, K., Takahashi, I., and Tanaka, K., 2014. "Genetic polymorphisms in japanese fragrant landraces and novel fragrant allele domesticated in northern Japan." Breeding Science, vol. 64, pp. 115-124.

[8] He, Y., Wang, S., and Ding, Y., 2013. "Identification of novel glutelin subunits and a comparison of glutelin composition between japonica and indica rice (Oryza sativa, L.) " Journal of Cereal Science, vol. 57, pp. 362-371.

[9] Peng, B., Pang, R. H., and Sun, Y. F., 2016. "Research progress in starch synthesis and its regulation in rice seeds." Acta Agriculturae Jiangxi, vol. 28, pp. 15-21.

[10] Hyun, D. Y., Lee, G. A., and Kang, M. J., 2015. "Development of low-temperature germinability markers for evaluation of rice (oryza sativa, 1.) germplasm." Molecular Breeding, vol. 34, pp. 1-16.

[11] Duan, M. and Sun, S. S., 2005. "Profiling the expression of genes controlling rice grain quality." Plant Molecular Biology, vol. 59, pp. 165-178.

[12] Cao, Z. Z., Zhang, Q. F., and Wei, K. S., 2012. "Response of some key enzyme activities involved in nitrogen metabolism to high temperature at filling stage and its relation to storage protein accu-mulation in rice grain." Acta Agronomica Sinica, vol. 38, pp. 99-106.

[13] Yang, F., Chen, Y., and Tong, C., 2014. "Association mapping of starch physicochemical properties with starch synthesis-related gene markers in non-waxy rice (Oryza sativa, L.)." Molecular Breeding, vol. 34, pp. 1747-1763.

[14] Jiang, L., Yu, X., and Qi, X., 2013. "Multigene engineering of starch biosynthesis in maize endosperm increases the total starch content and the proportion of amylose." Transgenic Research, vol. 22, p. 1133.

[15] Zhou, W., Wang, X., and Zhou, D., 2017. "Overexpression of the 16-kda $\alpha$-amylase/trypsin inhibitor rag2 improves grain yield and quality of rice." Plant Biotechnology Journal, vol. 15, p. 568.

[16] Wilson, S. A. and Roberts, S. C., 2014. "Metabolic engineering approaches for production of biochemicals in food and medicinal plants." Current Opinion in Biotechnology, vol. 26, p. 174.

[17] Hansson, S. O. and Joelsson, K., 2013. "Crop biotechnology for the environment." Journal of Agricultural \& Environmental Ethics, vol. 26, pp. 759-770.

[18] Yu, H. and Wang, T., 2016. "Proteomic dissection of endosperm starch granule associated proteins reveals a network coordinating starch biosynthesis and amino acid metabolism and glycolysis in rice endosperms." Frontiers in Plant Science, vol. 7, p. 707.

[19] Wang, J. C., Xu, H., and Zhu , Y., 2013. "Osbzip58, a basic leucine zipper transcription factor, regulates starch biosynthesis in rice endosperm." Journal of Experimental Botany, vol. 64, pp. 3453-3466.

[20] Peng, B., Sun, Y. F., and Pang, R. H., 2016. "Research progress of amino acid transporters in plants." Chinese Journal of Tropical Crops, vol. 37, pp. 1238-1243.

[21] Osuji, G. O., Duffus, E., and Johnson, P., 2015. "Enhancement of the essential amino acid composition of food crop proteins through biotechnology." American Journal of Plant Sciences vol. 6, pp. 3091-3108.

[22] Ufaz, S. and Galili, G., 2008. "Improving the content of essential amino acids in crop plants, Goals and opportunities." Plant Physiology, vol. 147, pp. 954-961. 
[23] Long, X., Liu, Q., and Chan, M., 2013. "Metabolic engineering and profiling of rice with increased lysine." Plant Biotechnology Journal, vol. 11, pp. 490-501.

[24] Azevedo, R. A. and Arruda, P., 2010. "High-lysine maize: the key discoveries that have made it possible." Amino Acids, vol. 39, pp. 979-989.

[25] Vidal, L. V. O., Xavier, T. O., and Michelato, M., 2015. "Apparent protein and energy digestibility and amino acid availability of corn and co-products in extruded diets for nile tilapia, oreochromis niloticus." Journal of the World Aquaculture Society, vol. 46, pp. 183-190.

[26] Galili, G. and Amir, R., 2013. "Fortifying plants with the essential amino acids lysine and methionine to improve nutritional quality." Plant Biotechnology Journal, vol. 11, pp. 211-222.

[27] Le, D. T., Chu, H. D., and Le, N. Q., 2016. "Improving nutritional quality of plant proteins through genetic engineering." Current Genomics, vol. 17, pp. 220-229.

[28] Yang, Q., Zhang, C., and Chan, M., 2016. "Biofortification of rice with the essential amino acid lysine: Molecular characterization, nutritional evaluation, and field performance." Journal of Experimental Botany, vol. 67, pp. 4285-4296.

[29] Kawakatsu, T. and Takaiwa, F., 2010. "Cereal seed storage protein synthesis, Fundamental processes for recombinant protein production in cereal grains." Plant Biotechnology Journal, vol. 8, pp. 939-953.

[30] Liu, X., Zhang, C., and Wang, X., 2016. "Development of high-lysine rice via endosperm-specific expression of a foreign lysine rich protein, gene." BMC Plant Biology, vol. 16, pp. 1-13.

[31] Wong, H. W., Liu, Q., and Sun, S. S., 2015. "Biofortification of rice with lysine using endogenous histones." Plant Molecular Biology, vol. 87, pp. 235-248.

[32] Monirujjaman, M. and Ferdouse, A., 2014. "Metabolic and physiological roles of branched-chain amino acids." Molecular Biology,,

[33] Birla, D. S., Malik, K., and Sainger, M., 2017. "Progress and challenges in improving the nutritional quality of rice (oryza sativa 1.)." Critical Reviews in Food Science and Nutrition, vol. 57, pp. 2455-2481.

[34] Zhu, X. and Galili, G., 2003. "Increased lysine synthesis coupled with a knockout of its catabolism synergistically boosts lysine content and also transregulates the metabolism of other amino acids in arabidopsis seeds." Plant Cell, vol. 15, pp. 845-853.

[35] Wakasa, K., Hasegawa, H., and Nemoto, H., 2006. "High-level tryptophan accumulation in seeds of transgenic rice and its limited effects on agronomic traits and seed metabolite profile." Journal of Experimental Botany, vol. 57, pp. 3069-3078.

[36] Tan, H., Liang, W., and Hu, J., 2012. "MTR1,Encodes a secretory fasciclin glycoprotein required for male reproductive development in rice." Developmental Cell, vol. 22, pp. 1127-1137.

[37] Wakasa, K. and Widholm, J. M., 1987. " A 5- Methyltryptophan resistant rice mutant, mtr1, selected in tissue culture." Theoretical and Applied Genetics, vol. 74, pp. 49-54.

[38] Wakasa, K. and Ishihara, A., 2010. "Metabolic engineering of the tryptophan and phenylalanine biosynthetic pathways in rice." Plant Biotechnology Journal, vol. 26, pp. 523-533.

[39] Schmidt, F., De, B. F. D., and Monteiro, F. A., 2013. "Sulfur limitation increases nitrate and amino acid pools in tropical forages." Crop and Pasture Science, vol. 64, pp. 1-5.

[40] Hooghe, P., Escamez, S., and Trouverie, J., 2013. "Sulphur limitation provokes physiological and leaf proteome changes in oilseed rape that lead to perturbation of sulphur, carbon and oxidative metabolisms." BMC Plant Biology, vol. 13, p. 23.

[41] Sílvia, T., Markus, W., and Beier, M. P., 2015. "Characterization of the serine acetyltransferase gene family of vitis viniferauncovers differences in regulation of OAS synthesis in woody plants." Frontiers in Plant Science, vol. 6, p. 74.

[42] Darabi, M., Izadidarbandi, A., and Masoudinejad, A., 2012. "Erratum to, Bioinformatics study of the 3hydroxy-3-methylglotaryl-coenzyme a reductase (hmgr) gene in gramineae." Molecular Biology Reports, vol. 39, pp. 8925-8935.

[43] Ying, G. E., Bai, X., and Cai, H., 2009. "Genetic engineering for enhancement of methionine in legume plants." Journal of Northeast Agricultural University, vol. 40, pp. 127-133.

[44] Shen, Y., Jia, Q. L., and Liu, M. Z., 2016. "Genome-wide characterization and phylogenetic and expression analyses of the caleosin gene family in soybean, common bean and barrel medic." Archives of Biological Sciences, vol. 68, pp. 575-585.

[45] Nguyen, H. C., Hoefgen, R., and Hesse, H., 2012. "Improving the nutritive value of rice seeds, Elevation of cysteine and methionine contents in rice plants by ectopic expression of a bacterial serine acetyltransferase." Journal of Experimental Botany, vol. 63, pp. 5991-6001.

[46] Xu, H., Wei, Y., and Zhu, Y., 2015. "Antisense suppression of lox3 gene expression in rice endosperm enhances seed longevity." Plant Biotechnology Journal, vol. 13, pp. 526-539.

[47] Goufo, P., Kratz, S., and Rosa, E. A. S., 2014. "Effect of elevated carbon dioxide concentration on rice quality: nutritive value, color, milling, cooking, and eating qualities." Cereal Chemistry, vol. 91, pp. 513521.

[48] Shao, Y. and Bao, J., 2015. "Polyphenols in whole rice grain: Genetic diversity and health benefits." Food Chemistry, vol. 180, pp. 86-97.

[49] Lado, J., Zacarías, L., and Rodrigo, M. J., 2016. "Regulation of carotenoid biosynthesis during fruit development." Subcellular Biochemistry, vol. 79, pp. 161-198. 
[50] Gayen, D., Ali, N., and Sarkar, S. N., 2015. "Down-regulation of lipoxygenase gene reduces degradation of carotenoids of golden rice during storage." Planta, vol. 242, pp. 353-363.

[51] Wysoczański, T., Sokoławysoczańska, E., and Pękala, J., 2016. "Omega-3 fatty acids and their role in central nervous system-a review." Current Medicinal Chemistry, vol. 23, pp. 816-831.

[52] Puaschitz, N. G., Strand, E., and Dierkes, J., 2015. "Dietary intake of saturated fat is not associated with risk of coronary events or mortality in patients with established coronary artery disease." Journal of Nutrition, vol. 145, pp. 299-305.

[53] Sun, Q., Jiang, L., and Qin, Z., 2013. "Characterization of three novel desaturases involved in the delta-6 desaturation pathways for polyunsaturated fatty acid biosynthesis from phytophthora infestans." Applied Microbiology \& Biotechnology, vol. 97, pp. 7689-7697.

[54] Liu, H. L., Yin, Z. J., and Xiao, L., 2012. "Identification and evaluation of $\omega-3$ fatty acid desaturase genes for hyperfortifying $\alpha$-linolenic acid in transgenic rice seed." Journal of Experimental Botany, vol. 36, pp. 3279-3287.

[55] Chodok, P., Cove, D. J., and Quatrano, R. S., 2012. "Metabolic engineering and oil supplementation of physcomitrella patens for activation of c22 polyunsaturated fatty acid production." Journal of the American Oil Chemists Society, vol. 89, pp. 465-476.

[56] Chen, Y., Meesapyodsuk, D., and Qiu, X., 2014. "Transgenic production of omega-3 very long chain polyunsaturated fatty acids in plants, Accomplishment and challenge." Biocatalysis and Agricultural Biotechnology, vol. 3, pp. 38-43.

[57] Cheah, T. W., Ismail, I., and Sidek, N. M., 2013. "Biosynthesis of very long polyunsaturated omega-3 and omega-6 fatty acids in transgenic japonica rice ,oryza sativa 1." Australian Journal of Crop Science, vol. 7, pp. 1227-1234.

[58] Bhattacharya, S., B., C., and L., K., 2014. "Heterologous expression of brassica juncea, microsomal $\omega-3$ desaturase gene (bjfad3) improves the nutritionally desirable $\omega-6, \Omega-3$ fatty acid ratio in rice bran oil." Plant Cell Tissue and Organ Culture, vol. 119, pp. 117-129.

[59] Liu, L., Waters, D. L., and Rose, T. J., 2013. "Phospholipids in rice: Significance in grain quality and health benefits." A Review J Food Chemistry, vol. 139, pp. 1133-1145.

[60] Choi, N., Lee, J. S., and Kwak, J., 2016. "Production of biodiesel from acid oil via a two-step enzymatic transesterification." Journal of Oleo Science, vol. 65, pp. 913-921.

[61] Yin, Z. J., Liu, H. L., and Dong, X. B., 2014. "Increasing $\alpha$-linolenic acid content in rice bran by embryospecific expression of $\omega 3 / \Delta 15$-desaturase gene." Molecular Breeding, vol. 33, pp. 987-996.

[62] Bogdanov, I. V., Shenkarev, Z. O., and Finkina, E. I., 2016. "A novel lipid transfer protein from the pea pisum sativum, Isolation, recombinant expression, solution structure, antifungal activity, lipid binding, and allergenic properties." BMC Plant Biology, vol. 16, p. 107.

[63] Wang, X., Zhou, W., and Lu, Z., 2015. "A lipid transfer protein, osltpl36, is essential for seed development and seed quality in rice." Plant Science, vol. 239, pp. 200-208.

[64] Headey, D., Hoddinott, J., and Ali, D., 2015. "The other asian enigma, Explaining the rapid reduction of undernutrition in Bangladesh." World Development, vol. 66, pp. 749-761.

[65] Pugliese, A., O'Callaghan, Y., and Tundis, R., 2014. "In vitro investigation of the bioaccessibility of carotenoids from raw, frozen and boiled red chili peppers ,capsicum annuum." European Journal of Nutrition, vol. 53, pp. 501-510.

[66] Gemmecker, S., Schaub, P., and Koschmieder, J., 2015. "Phytoene desaturase from oryza sativa, Oligomeric assembly, membrane association and preliminary 3d-analysis." PLOS ONE, vol. 10,

[67] Ye, X. D., Al-Babili, S., and Klöti, A., 2000. "Engineering the provitamin a ( $\beta$-carotene) biosynthetic pathway into (carotenoid-free) rice endosperm." Scienc, vol. 287, pp. 303-305.

[68] Ji, C. M., Shin, J. A., and Cho, J. W., 2013. "Nutritional evaluation of immature grains in two korean rice cultivars during maturation." Food Science and Biotechnology, vol. 22, pp. 903-908.

[69] Paine, J. A., Shipton, C. A., and Chaggar, S., 2005. "Improving the nutritional value of golden rice through increased pro-vitamin a content." Nature Biotechnology, vol. 23, pp. 482-487.

[70] Kriegerliszkay, A. and Feilke, K., 2016. "The dual role of the plastid terminal oxidase ptox, Between a protective and a pro-oxidant function." Frontiers in Plant Science, vol. 6, p. 1147.

[71] Hu, P., Wang, Y., and Przybyłowicz, W. J., 2015. "Elemental distribution by cryo-micro-pixe in the zinc and cadmium hyperaccumulator sedum plumbizincicola, grown naturally." Plant and Soil, vol. 388, pp. 267-282.

[72] Gerdes, S., Lermaortiz, C., and Frelin, O., 2012. "Plant b vitamin pathways and their compartmentation, A guide for the perplexed." Journal of Experimental Botany, vol. 63, pp. 5379-5395.

[73] Barbara, L. and Hendrik, K., 2013. "Compartmentation and complexation of metals in hyperaccumulator plants." Frontiers in Plant Science, vol. 4, p. 374.

[74] Dong, W., Stockwell, V. O., and Goyer, A., 2015. "Enhancement of thiamin content in arabidopsis thaliana by metabolic engineering." Plant and Cell Physiology, vol. 56, pp. 2285-2296.

[75] Bocobza, S., E., Malitsky, S., and Araújo, W. L., 2013. "Orchestration of thiamin biosynthesis and central metabolism by combined action of the thiamin pyrophosphate riboswitch and the circadian clock in arabidopsis." Plant Cell, vol. 25, pp. 288-307.

[76] Pourcel, L., Moulin, M., and Fitzpatrick, T. B., 2013. "Examining strategies to facilitate vitamin b1 biofortification of plants by genetic engineering." Frontiers in Plant Science, vol. 4, p. 160. 
[77] Ami, N., Bernstein, M., and Boucher, F., 2016. "Folate and neural tube defects, The role of supplements and food fortification." Paediatr Child Health, vol. 21, pp. 145-149.

[78] Arif, S., Khan, M. R., and Gardezi, S. D. A., 2016. "A novel hydroxymethyldihydropterin pyrophosphokinase-dihydropteroate synthase (hppk-dhps) gene from a nutraceutical plant seabuckthorn, involved in folate pathway is predominantly expressed in fruit tissue." International Journal of Agriculture and Biology, vol. 18, pp. 412-418.

[79] Macfarlane, A. J., Behan, N. A., and Matias, F. M., 2013. "Dietary folate does not significantly affect the intestinal microbiome, inflammation or tumorigenesis in azoxymethane-dextran sodium sulphate-treated mice." British Journal of Nutrition, vol. 109, pp. 630-638.

[80] Yue, H., Liang, Q., and Zhang, W., 2016. "A monoclonal antibody-based enzyme-linked immunosorbent assay for 5-formyltetrahydrofolate detection in maize kernels." Food Analytical Methods, vol. 9, pp. 1-8.

[81] Blancquaert, D., Van, D. J., and Storozhenko, S., 2013. "Rice folate enhancement through metabolic engineering has an impact on rice seed metabolism, but does not affect the expression of the endogenous folate biosynthesis genes." Plant Molecular Biology, vol. 83, pp. 329-349.

[82] Farré, G., Sudhakar, D., and Naqvi, S., 2012. "Transgenic rice grains expressing a heterologous $\rho-$ hydroxyphenylpyruvate dioxygenase shift tocopherol synthesis from the $\gamma$ to the $\alpha$ isoform without increasing absolute tocopherol levels." Transgenic Research, vol. 21, pp. 1093-1097.

[83] Wang, X., Song, Y., and Li, J., 2013. "High expression of tocochromanol biosynthesis genes increases the vitamin e level in a new line of giant embryo rice." Journal of Agricultural and Food Chemistry, vol. 61, pp. 5860-5869.

[84] Chen, Y., Liu, L., and Shen, Y., 2015. "Loss of function of the cytochrome p450 gene cyp78b5, causes giant embryos in rice." Plant Molecular Biology Reporter, vol. 33, pp. 69-83.

[85] Hwang, J. E., Ahn, J. W., and Kwon, S. J., 2014. "Selection and molecular characterization of a high tocopherol accumulation rice mutant line induced by gamma irradiation." Molecular Biology Reports, vol. 41, pp. 7671-7681.

[86] Jiang, J., Jia, H., and Feng, G., 2016. "Overexpression of medicago sativa tmt elevates the $\alpha$-tocopherol content in arabidopsis seeds, alfalfa leaves, and delays dark-induced leaf senescence." Plant Science, vol. 249, pp. 93-104.

[87] Naidu, K. A. and Vitamin, C., 2003. "In human health and disease is still a mystery." Nutrition Journal, vol. 2, pp. 7-7.

[88] Zhang, G. Y., Liu, R. R., ., and Zhang, C. Q., 2015. "Manipulation of the rice 1-galactose pathway, Evaluation of the effects of transgene overexpression on ascorbate accumulation and abiotic stress tolerance." PLOS ONE, vol. 10,

[89] Höller, S., Ueda, Y., and Wu, L., 2015. "Ascorbate biosynthesis and its involvement in stress tolerance and plant development in rice, Oryza sativa L." Plant Molecular Biology, vol. 88, pp. 545-560.

[90] Biselli, C., Bagnaresi, P., and Cavalluzzo, D., 2015. "Deep sequencing transcriptional fingerprinting of rice kernels for dissecting grain quality traits." BMC Genomics, vol. 16, p. 1091.

[91] Farré, G., Blancquaert, D., and Capell, T., 2014. "Engineering complex metabolic pathways in plants." Annual Review of Plant Biology, vol. 65, pp. 187-223.

[92] Zhang, Z., Zhao, H., and Tang, J., 2014. "A proteomic study on molecular mechanism of poor grain-filling of rice, oryza sativa 1, inferior spikelets." PLOS ONE, vol. 9, p. 89140.

[93] Degenkolbe, T., Do, P. T., and Kopka, J., 2013. "Identification of drought tolerance markers in a diverse population of rice cultivars by expression and metabolite profiling." PLoS ONE, vol. 8, p. e63637.

[94] Maruyama, K. and Yamaguchi-Shinozaki, K., 2014. "Integrated analysis of the effects of cold and dehydration on rice metabolites, phytohormones, and gene transcripts." Plant Physiology, vol. 164, pp. 1759-1771.

[95] Samanta, M. K., Dey, A., and Gayen, S., 2016. "Crispr/cas9, An advanced tool for editing plant genomes." Transgenic Research, vol. 25, pp. 561-573.

[96] Liu, H., Ding, Y., and Zhou, Y., 2017. "Crispr-p 2.0, An improved crispr-cas9 tool for genome editing in plants." Molecular Plant, vol. 10, pp. 530-532.

[97] Xu, R., Wei, P., and Yang, J., 2017. "Use of crispr/cas genome editing technology for targeted mutagenesis in rice." Methods in Molecular Biology, vol. 1498, pp. 33-40.

[98] Li, X., Zhou, W., and Ren, Y., 2017. "High-efficiency breeding of early-maturing rice cultivars via crispr/cas9- mediated genome editing." Journal of Genetics and Genomics, vol. 44, pp. 175-178.

[99] Sun, Y., Jiao, G., and Liu, Z., 2017. "Generation of high-amylose rice through crispr/cas9-mediated targeted mutagenesis of starch branching enzymes." Frontiers in Plant Science, vol. 8, p. 298. 